\title{
New infrastructure for studies of transmutation and fast systems concepts
}

\author{
Fabio Panza ${ }^{1, \mathrm{a}}$, Gabriele Firpo ${ }^{2}$, Guglielmo Lomonaco ${ }^{1,3}$, Mikhail Osipenko ${ }^{1}$, Giovanni Ricco ${ }^{1,4}$, Marco Ripani ${ }^{1,4}$, Paolo \\ Saracco $^{1}$ and Carlo Maria Viberti $^{2}$ \\ ${ }^{l}$ Istituto Nazionale di Fisica Nucleare - Sezione di Genova, V. Dodecaneso, 33 - 16146 Genova (Italy) \\ ${ }^{2}$ Ansaldo Nucleare C. so F.M. Perrone, 2516152 Genova (Italy) \\ ${ }^{3}$ GeNERG DIME/TEC - University of Genova, V. all'Opera Pia, 15/A - 16145 Genova (Italy) \\ ${ }^{4}$ Centro Fermi - Compendio del Viminale - Piazza del Viminale 1 - 00184 Rome (Italy)
}

\begin{abstract}
In this work we report initial studies on a low power Accelerator-Driven System as a possible experimental facility for the measurement of relevant integral nuclear quantities. In particular, we performed Monte Carlo simulations of minor actinides and fission products irradiation and estimated the fission rate within fission chambers in the reactor core and the reflector, in order to evaluate the transmutation rates and the measurement sensitivity. We also performed a photo-peak analysis of available experimental data from a research reactor, in order to estimate the expected sensitivity of this analysis method on the irradiation of samples in the ADS considered.
\end{abstract}

\section{Introduction}

The scope of this work is the design optimization, via Monte Carlo simulations (with the MCNP6 [1] and MCB [2] codes), of a subcritical system to perform integral measurements on transmutation processes. Such a system may be an intermediate step between the zero-power ADS Guinevère [3] and the future Myrrha [4].

In this initial study we have considered:

- Actinide and FP pellets inserted into the core at two different positions, studying the burn-up in terms of variation of the initial isotopes and appearance of fission products

- Fission chambers with fissile or fissionable isotope depositions, studying the fission rate for a specific setup

- Photo-peak analysis of irradiated samples, as a method to determine the integral fission based on the appearance of specific FP and to determine the integral capture on U-238 based on the appearance of Np-239

\section{ADS description}

The geometry of the subcritical core is derived from [5], where the accelerator driver is a $70 \mathrm{MeV}$ proton beam generated by a commercial cyclotron. With respect to the original studies on transmutation capabilities of above described machine [6], we increased the number of Fuel Assemblies (FAs) from 60 to 110 (increasing the Lead reflector radius accordingly) and changed the fuel from $\mathrm{UO}_{2}$ with $20 \%$ enrichment to the Superphenix MOX composition [7],

\footnotetext{
${ }^{\mathrm{a}}$ Corresponding author: Fabio.Panza@ge.infn.it
}

obtaining a $\mathrm{k}_{\text {eff }}$ around 0.97 and a thermal power around $430 \mathrm{~kW}$.

All the MCNP simulations reported here have been performed using a measured source spectrum obtained in a dedicated experiment [8]. We assumed a proton beam with $1 \mathrm{~mA}$ current, corresponding approximately to a total rate of neutron production from the beryllium target of $7.6 \cdot 10^{14} \mathrm{n} / \mathrm{s}$. In Figure 1, the configuration and the selected irradiation positions in the xy plane are shown. The irradiation positions characteristics are reported in Table 1.

Table 1. Neutron fluxes in the 110 FAs configuration for the two considered positions (errors are statistical).

\begin{tabular}{|c|c|}
\hline Position & Integral Flux $\left(\mathbf{n} / \mathbf{c m}^{\mathbf{2}} / \mathbf{s}\right)$ \\
\hline $\mathbf{A}$ & $(1.53 \pm 0.01) \mathrm{E}+13$ \\
\hline B & $(4.82 \pm 0.01) \mathrm{E}+11$ \\
\hline
\end{tabular}

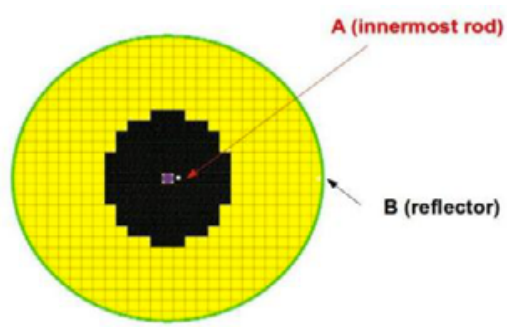

Figure 1: 110 FAs configuration plot in xy plane with the irradiation positions: Position $\mathrm{A}$ is close to the source and $\mathrm{B}$ at the reflector periphery. Purple: Beryllium target; black: reactor core; yellow: Lead reflector; green: Stainless Steel. 
The energy spectrum and axial distributions in the two positions close to the source (A) and in the reflector periphery (B) are plotted in Figure 2 and in Figure 3 respectively.

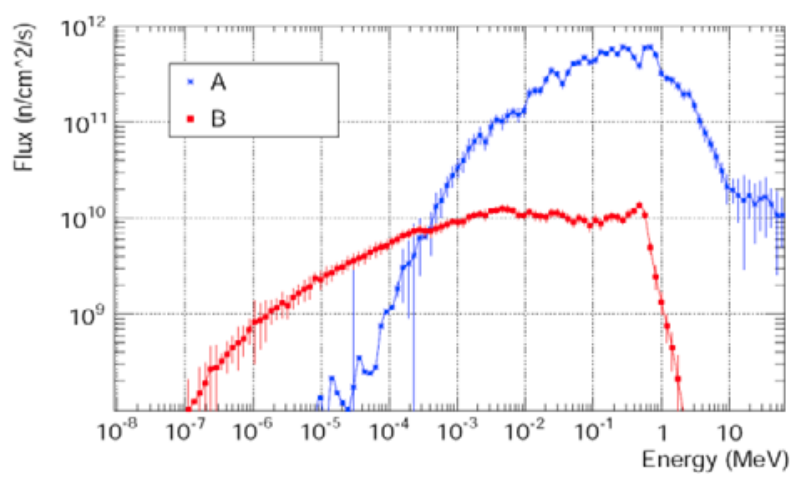

Figure 2: Energy flux distributions in the two positions near the source (A) and in the reflector (B).

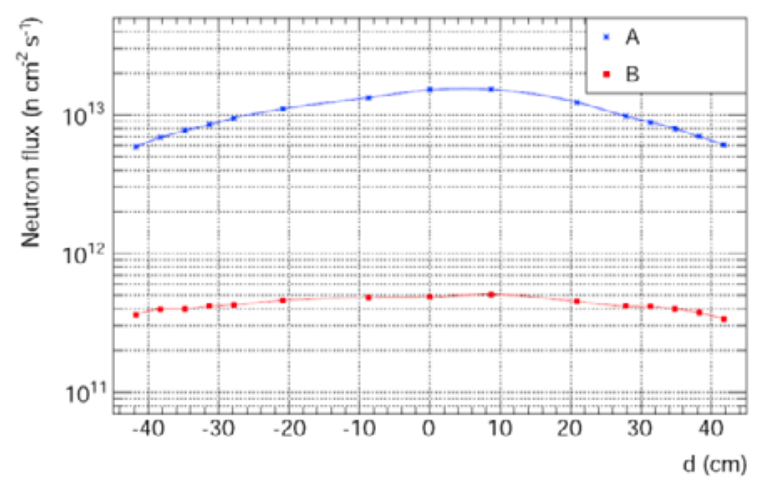

Figure 3: Axial flux distributions in the two positions near the source (A) and in the reflector (B): 0 represents the core mid-plane.

\section{Minor actinides and FP irradiation}

One of the purpose of these studies is to show how a low-power ADS can be used for integral measurements of nuclear properties relevant to future fast systems. Therefore, we studied the effect of specific irradiations by using the MCB code, in order to investigate the transmutation rate of selected nuclides. In particular, we simulated the irradiation of pellets of Minor Actinides (MA), Np-237, Am-241, Cm-244, as well as Medium Lived Fission Products (MLFP), Sr-90, Cs137 and Long Lived Fission Products (LLFP), Se-79, Tc-99, Pd-107, Sn-126, I-129, Cs-135. The irradiations were performed by introducing in the simulations a dedicated irradiation channel near the neutron source in the equatorial position, in order to have the highest flux intensity (see Figure 1, position A). The dimensions of the pellet were $0.357 \mathrm{~cm}$ radius and $2 \mathrm{~cm}$ thickness. The evolution of the pellet composition was calculated by considering one year of irradiation (including the natural decay) in the ADS, or one year of pure decay, then comparing the two final compositions. The difference between the mass after $1 \mathrm{y}$ of decay and $1 \mathrm{y}$ of irradiation (i.e., $\boldsymbol{\Delta M}$ ) , with respect to the initial pellet mass (i.e, $\boldsymbol{M})$ are reported in the Tables 2 to 4 .

Table 2. Results for the irradiation of MA pellets.

\begin{tabular}{|c|c|}
\hline Nuclide & $\boldsymbol{M M} / \boldsymbol{M}(\boldsymbol{\%})$ \\
\hline Np-237 & $6.35 \mathrm{E}-01$ \\
\hline Am-241 & $6.90 \mathrm{E}-02$ \\
\hline Cm-244 & $4.68 \mathrm{E}-02$ \\
\hline
\end{tabular}

Table 3. Results for the irradiation of MLFP pellets.

\begin{tabular}{|c|c|}
\hline Nuclide & $\boldsymbol{\Delta M} / \boldsymbol{M}(\boldsymbol{\%})$ \\
\hline Sr-90 & $1.22 \mathrm{E}-03$ \\
\hline Cs-137 & $1.14 \mathrm{E}-03$ \\
\hline
\end{tabular}

Table 4. Results for the irradiation of LLFP pellets.

\begin{tabular}{|c|c|}
\hline Nuclide & $\boldsymbol{A M} / \boldsymbol{M}(\boldsymbol{\%})$ \\
\hline Se-79 & $9.28 \mathrm{E}-03$ \\
\hline Tc-99 & $1.53 \mathrm{E}-02$ \\
\hline Pd-107 & $2.39 \mathrm{E}-02$ \\
\hline $\mathbf{I - 1 2 9}$ & $9.25 \mathrm{E}-03$ \\
\hline Cs-135 & $2.04 \mathrm{E}-03$ \\
\hline
\end{tabular}

As we can see from the tables, the contribution of the irradiation to the transmutation, obtained by subtracting the variation due to the decay, is about $10^{-1}$ $\%$ to $10^{-2} \%$ for MA and about $10^{-2} \%$ for LLFP.

The transmutation contribution for MLFP and for some LLFP is about $10^{-3} \%$, which can be easily understood if we consider their capture cross sections. In some of these cases, a softer neutron spectrum may be necessary, which may be the subject of specific optimizations of the system.

\section{Fission chambers}

We also performed an evaluation of the fission rate achievable in Fission Chambers (FC) with different depositions (U-235, U-238, Np-237, Pu-239, Pu-241, Am-241), for each of them assuming a typical mass $\mathrm{m}=10 \mu \mathrm{g}$ and assuming $100 \%$ detection efficiency. In the simulation the FC were placed in either of the two above-mentioned positions ( $\mathrm{A}$ and $\mathrm{B}$ in Figure. 1) within the core and in the Lead reflector respectively. The results are reported in Table 5. 
Table 5. Fission rates $\mathrm{R}$ for fission chambers with different depositions. A and B are the measurement positions close to the source and in the reflector, respectively (see Figure 1).

\begin{tabular}{|c|c|c|}
\hline Material & $\mathbf{R}$ (fiss/s) in A & R (fiss/s) in B \\
\hline $\mathbf{U - 2 3 5}$ & $(7.15 \pm 0.20) \mathrm{E}+05$ & $(3.19 \pm 0.63) \mathrm{E}+05$ \\
\hline $\mathbf{U - 2 3 8}$ & $(2.51 \pm 0.03) \mathrm{E}+04$ & $(2.37 \pm 0.25) \mathrm{E}+01$ \\
\hline Np-237 & $(6.80 \pm 0.08) \mathrm{E}+05$ & $(1.47 \pm 0.12) \mathrm{E}+05$ \\
\hline $\mathbf{P u - 2 3 9}$ & $(9.09 \pm 0.10) \mathrm{E}+05$ & $(2.45 \pm 0.12) \mathrm{E}+05$ \\
\hline $\mathbf{P u - 2 4 1}$ & $(2.01 \pm 0.21) \mathrm{E}+05$ & $(1.62 \pm 0.42) \mathrm{E}+03$ \\
\hline Am-241 & $(1.60 \pm 0.11) \mathrm{E}+05$ & $(1.01 \pm 0.07) \mathrm{E}+03$ \\
\hline
\end{tabular}

These simulations give us an idea of the integral measurements of fission rates of U-235, U-238, Np237, Pu-239, Pu-241 and Am-241 in two different reactor positions, therefore with neutron fluxes that differ in both intensity and shape. In particular, with the assumed mass, which appears relatively modest, the counting rates obtained are high enough that high precision measurements can be performed within a few seconds to within several minutes (e.g., in the case of $\mathrm{U}-238$ in position $\mathrm{B})$.

\section{Gamma spectroscopy}

As part of the integral measurements offered by the proposed ADS facility, we studied a possible method to experimentally estimate the MOX fuel burn-up. We explored the possibility to exploit gamma lines from FP appearing after irradiation. As a practical example, we have analyzed the gamma spectrum measured from a natural Uranium pellet irradiated for 6 hours at 250 $\mathrm{W}$ in the central channel of the TRIGA MARK II facility, a thermal research reactor of the LENA laboratory (University of Pavia).

An HPGe detector yielded the gamma spectrum that was analyzed with the Gamma Vision ${ }^{\circledR}$ code by ORTEC $^{(2}$. Our purpose was to evaluate the sensitivity and the systematic uncertainty of this measurement. To get a feeling of the systematic uncertainty, we compared the results of the Gamma Vision ${ }^{\circledR}$ code to manual fits performed by means of the ROOT analysis framework [9]. In Figure 4 we report the gamma spectrum of the natural Uranium pellet after the irradiation.

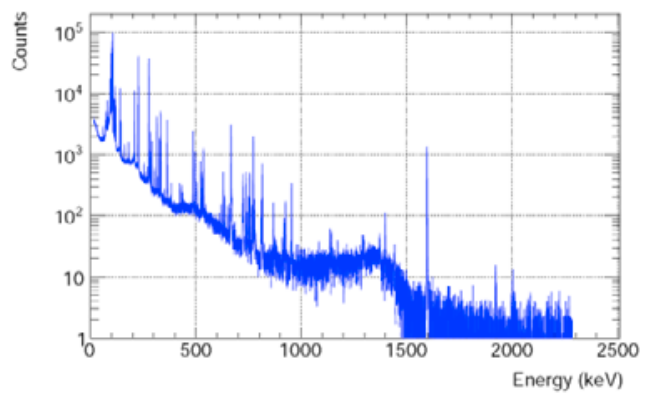

Figure 4: Gamma spectrum measured from a natural Uranium pellet irradiated for 6 hours at $250 \mathrm{~W}$ in the central channel of the TRIGA MARK II facility, a thermal research reactor of the LENA laboratory (University of Pavia).

We have considered some specific fission products featuring well-isolated and easily identifiable photopeaks (La-140, Mo-99, Tc-99m) in the Gamma Vision ${ }^{\circledR}$ program and evaluated the activity of each single peak. To calculate the activity of each nuclide, we corrected the number of counts $\mathrm{N}$ in each peak according to the formula below, using five factors that take into account the decay during the irradiation and between the end of irradiation and the startup of counting (TDC), the total counting live time (LT), the branching ratio into that particular peak (BR), the detector efficiency $(\varepsilon)$, and the self-absorption coefficient $\left(\mathrm{A}_{\mathrm{c}}\right)$ [10], according to the following formula

$$
A=\frac{N \cdot T D C}{L T \cdot B R \cdot \varepsilon \cdot A_{C}}
$$

The obtained numbers of counts for the selected peaks are reported in table Table 6 .

Then, as an alternative analysis, we have considered the region around each peak and we have fitted the peak+background with a gaussian and a linear function, as shown in the following formula,

$$
N(E)=a \cdot e^{-\frac{(E-b)^{2}}{2 c^{2}}}+d \cdot E+e
$$

with five parameters, in order to obtain the counting rate in the signal region using the ROOT analysis package.

Obviously, in order to minimize the statistical uncertainties, we have considered the most populated (but well isolated) peaks for each isotope.

The fitted photo-peaks for La-140, Mo-99, Tc-99m, are shown in figures 5 to 7 respectively,

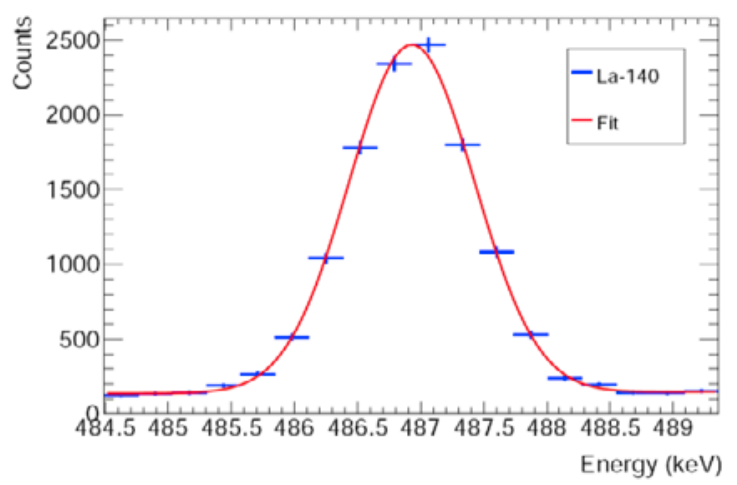

Figure. 5. The $487.03 \mathrm{keV}$ photo-peak of La-140, along with the ROOT fit. 


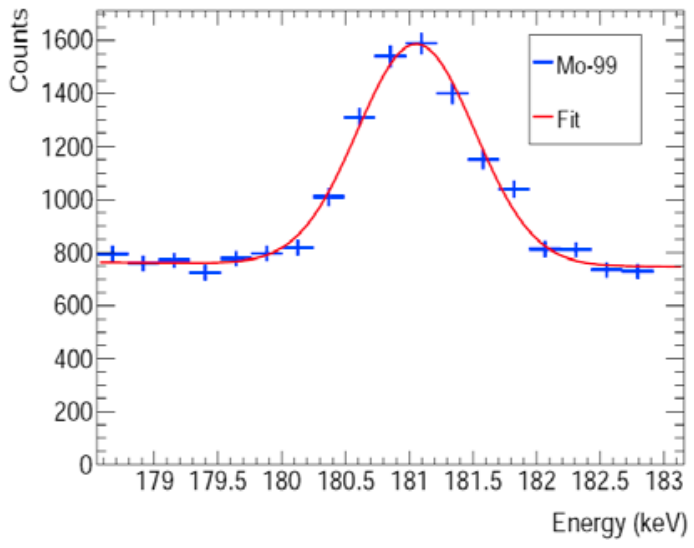

Figure. 6. The $181.09 \mathrm{keV}$ photo-peak of Mo-99, along with the ROOT fit.

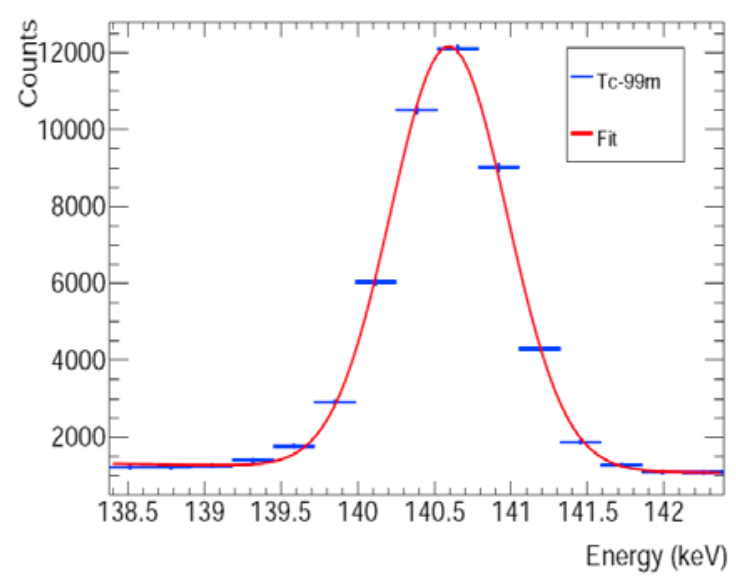

Figure. 7. The $140.51 \mathrm{keV}$ photo-peak for Tc-99m, along with the ROOT fit.

Any difference between the peak area obtained with ROOT and Gamma Vision ${ }^{\text {beyond statistical }}$ uncertainties would be interpreted as systematic uncertainty. However, in the particular cases considered, we found the two independent results to be statistically compatible. The comparison between the two methods is reported in Table 6.

Table 6: Comparison of the ROOT fits and the GammaVision program for the areas of the photo-peaks of all three isotopes considered.

\begin{tabular}{|c|c|c|}
\hline Nuclide & $\begin{array}{c}\text { Counts. from } \\
\text { ROOT }\end{array}$ & Counts from . GV \\
\hline La-140 & $10667 \pm 17$ & $10592 \pm 123$ \\
\hline Mo-99 & $3921 \pm 129$ & $3704 \pm 145$ \\
\hline Tc-99m & $39409 \pm 90$ & $39001 \pm 256$ \\
\hline
\end{tabular}

Obviously, longer measurements would lead to smaller statistical errors, which could reveal a systematic difference between the two methods.
Once the activity has been calculated, it is possible to perform a fuel burn-up evaluation based on the appearance of the above-mentioned FP. In the present example where we analyzed the data from the TRIGA, we evaluated the burn-up of the U-235 contained in the natural uranium fuel. In the case of the ADS with MOX fuel, the method can be applied to evaluate the main U-235 and Pu-239 burn-up together (obviously $\mathrm{Pu}-239$ is both created and destroyed; here we only measure the amount of $\mathrm{Pu}-239$ that underwent fission). Considering the production of a specific fission product, knowing its corresponding yield in the fission process $\left(\mathrm{Y}_{\mathrm{f}}\right)$ and its activity $(\mathrm{A})$, the burn-up, or in other words the number of fissions, can be determined using the following formula:

$$
n_{F}=\frac{A}{\lambda \cdot Y_{f}}
$$

where $\lambda$ is the nuclide decay constant. In Table 7 , we report the fuel burn-up evaluated from different fission fragments.

Table 7: Calculated activities using ROOT fits for the three selected photo-peaks and corresponding evaluation of the U235 burn-up in the data from the TRIGA reactor in Pavia.

\begin{tabular}{|c|c|c|}
\hline Nuclide & Activity (Bq) & $\begin{array}{c}\text { U-235 Fiss. } \\
\text { mass (g) }\end{array}$ \\
\hline La-140 & $(3.92 \pm 0.05) \mathrm{E}+04$ & $(5.18 \pm 0.04) \mathrm{E}-11$ \\
\hline Mo-99 & $(2.29 \pm 0.08) \mathrm{E}+04$ & $(5.06 \pm 0.19) \mathrm{E}-11$ \\
\hline Tc-99m & $(2.30 \pm 0.06) \mathrm{E}+05$ & $(5.20 \pm 0.04) \mathrm{E}-11$ \\
\hline
\end{tabular}

These results has been compared with the analytic formula which gave a U-235 fissioned mass of 5.14E$11 \mathrm{~g}$ by considering an effective fission cross section of $102 \mathrm{~b}$ for the central channel of the TRIGA MARK II reactor [10]. The results from the analysis of the three isotopes nicely agree with each other and with the expected value from the effective fission cross section. In order to find a possible application of this method to a MOX fuel (in which the fission fragments come mainly from U-238, U-235 and $\mathrm{Pu}-239$ ), we propose to consider three different fission product activity measurements, thereby solving a system of three equations in three variables to distinguish the contributions from the different nuclides as reported below.

$$
\begin{aligned}
& N_{F P 1}=Y_{U-235}^{F P 1} \cdot N_{U}-235+Y_{U-238}^{F P 1} \cdot N_{U-238}+Y_{P u-239}^{F P 1} \cdot N_{P u}-239^{N} F P 1 \\
& \left\{N_{F P 2}=Y_{U-235^{*}}^{F P 2} N_{U-235}+Y_{U-238^{*}}^{F P 2} N_{U}-238^{+}+Y_{P u-239^{*}}^{F P} N_{P u}-239^{N} F P 2\right. \\
& N_{F P 2}=Y_{U-235}^{F P 3} N_{U-235}+Y_{U-238}^{F P 3} \cdot N_{U}-238+Y_{P u-239}^{F P 3} \cdot N_{P u-239^{N}} F P 3
\end{aligned}
$$


To estimate the irradiation time necessary to obtain the same quantity, for example, of La-140 from U-238, in the ADS as in the TRIGA, we have used a Bateman equation. The resulting irradiation time is about $1000 \mathrm{~s}$. On the same line, we have also fitted the Np-239 photo-peak from the TRIGA data in order to evaluate the integral capture on U-238. In this case, the ROOT fit and the Gamma Vision ${ }^{\circledR}$ program turn out to be statistically incompatible, with a systematic difference of about $4 \%$ : the results are shown in Table 8 .

Table 8: Comparison of the ROOT fits and the Gamma Vision ${ }^{\circledR}$ program for the area of the photo-peak of Np-239.

\begin{tabular}{|l|c|c|c|}
\hline Nuclide & $\begin{array}{c}\text { Counts } \\
\text { from. ROOT }\end{array}$ & $\begin{array}{c}\text { Counts from . } \\
\text { GV }\end{array}$ & Diff. (\%) \\
\hline Np-239 & $139551 \pm 141$ & $144535 \pm 380$ & $3.95 \pm 0.01$ \\
\hline
\end{tabular}

For the Neptunium-239 activity, the result is $(3.72 \pm 0.01) \mathrm{E}+05 \mathrm{~Bq}$ (from ROOT analysis), to be compared with the numerical calculation performed by using an effective cross section in [10], which gives $3.70 \mathrm{E}+05 \mathrm{~Bq}$.

The fitted Np-239 photo-peak is shown in Figure 8.

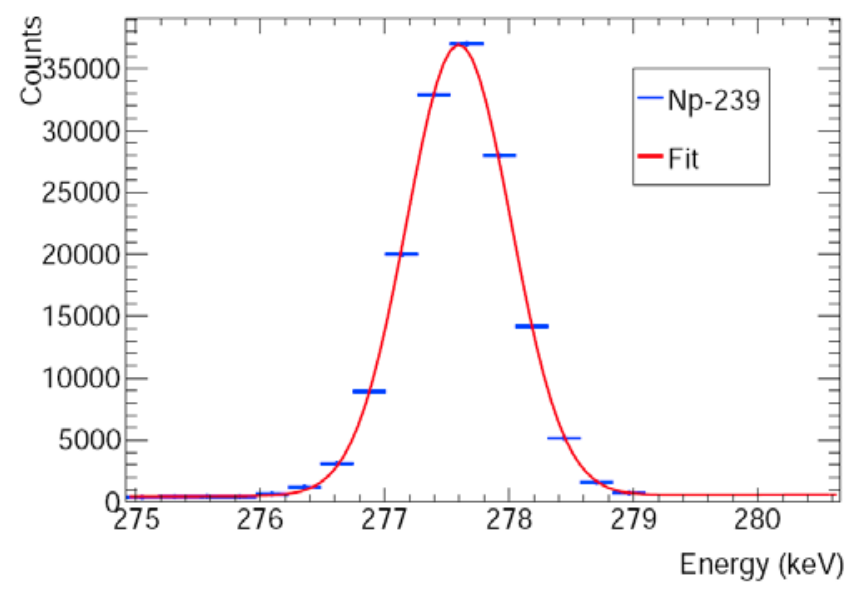

Figure. 8. The $277.60 \mathrm{keV}$ photo-peak for Np-239, along with the ROOT fit.

In order to estimate the sensitivity of this method applied to the burn-up evaluation in a MOX fuel pellet placed in our reference ADS (same dimensions as the actinide pellets of section 4 , irradiation time: $1 \mathrm{y}$ ), it is useful to calculate the Minimum Detectable Activity (MDA) in the regions of the spectra where some MA photo-peaks will be present. The MDA at 95\% CL is given by [5]:

$$
M D A=\frac{2.71+4.65 \cdot \sigma_{b g}}{L T \cdot \epsilon \cdot B R}
$$

where:

- $\sigma_{b g}$ is the poissonian background standard deviation

- LT is the live time of the data acquisition

$-\varepsilon$ is the detector efficiency

- BR is the peak branching ratio

The results are reported in table 9, where we report the simulated activity for $\mathrm{Am}$ and $\mathrm{Cm}$ isotopes, along with the corresponding MDA. To calculate the MDA, it is necessary to make some assumption about the counts from the background in a specific measurement time. Therefore, as an indicative estimate, we assumed the same spectrum measured in the TRIGA of Pavia and the same total measurement live time. We see that Am241, Am-243 and Cm-243 should all be detectable in a live-time of about 200 s. For the Cm-244, a much longer measurement time would be needed, in the order of a couple of days, since the MDA is significantly larger than the activity produced in the ADS in 1y. Finally, the $\mathrm{Cm}-245$ is produced in a negligible and non-measurable amount.

Table 9: Sensitivity of the HPGe detector to MA photopeaks

\begin{tabular}{|c|c|c|c|c|}
\hline Nuclide & $\begin{array}{c}\text { Gamma } \\
\text { Energy } \\
\text { (keV) }\end{array}$ & $\boldsymbol{\varepsilon}$ (\%) & $\begin{array}{c}\text { A (Bq) } \\
\text { after 1y } \\
\text { irradiatio } \\
\text { n }\end{array}$ & $\begin{array}{c}\text { MDA } \\
\text { (Bq) }\end{array}$ \\
\hline Am-241 & 59.54 & 3.9 & $6.91 \mathrm{E}+08$ & 226.25 \\
\hline $\mathbf{A m - 2 4 3}$ & 74.76 & 5.4 & $7.18 \mathrm{E}+04$ & 407.74 \\
\hline $\mathbf{C m}-\mathbf{2 4 3}$ & 276.68 & 9.4 & $1.31 \mathrm{E}+03$ & 133.91 \\
\hline $\mathbf{C m}-\mathbf{2 4 4}$ & 42.82 & 2.06 & $6.05 \mathrm{E}+03$ & $1.58 \mathrm{E}+05$ \\
\hline $\mathbf{C m}-\mathbf{2 4 5}$ & 130.05 & 15.39 & $9.97 \mathrm{E}-04$ & 590.76 \\
\hline
\end{tabular}

\section{Conclusions}

We simulated an Accelerator Driven System comprising a proton cyclotron with $70 \mathrm{MeV}$ beam energy and $1 \mathrm{~mA}$ beam current. The core is made up by 110 fuel assemblies, each hosting 81 fuel rods with a Superphenix-type MOX. By simulating the insertion into the core (innermost rod) of various MA and FP pellets, we studied the variation of the initial isotopes, the appearance of fission products for the Actinides and the transmutation by capture of the FP. We observed that the percentage transmutation with respect to natural decay is about $10^{-2}$ to $10^{-1} \%$ for MA, while for LLFP and MLFP is about $10^{-3}$ to $10^{-2} \%$.

We simulated the rate of fission chambers with fissile or fissionable isotope (U-235, U-238, Np-237, Pu-239, $\mathrm{Pu}-241$, Am-241) depositions, considering two irradiation positions: close to the source and in the lead reflector periphery. Our results indicate that measurements of high precision can be performed within a few seconds to within several minutes.

We performed a photo-peak analysis of an irradiated natural Uranium sample (data taken at the TRIGA reactor of Pavia), as a method to determine the integral fission based on the appearance of specific FP and to determine the integral capture on U-238 based on the 
appearance of Np-239. The analysis has been performed by evaluating the activity of the appeared fission product photo-peaks for La-140, Mo-99 and Tc$99 \mathrm{~m}$. We compared results for the peak area obtained from the Gamma Vision ${ }^{\circledR}$ program and from fits of the photo-peaks considered, performed with the ROOT analysis tool. The results are compatible within the statistical uncertainties, except for the U-238 capture where a $4 \%$ discrepancy between the two analyses was observed. The methodology presented can be applied also to the study of the integral fission in the MOX fuel, assuming the fission products to come mainly from U-235, U-238 and Pu-239, by considering the activity of at least three different isotopes and by solving a system of three equations with three variables (the number of fissioned nuclides).

\section{Acknowledgements}

The research leading to these results has received funding from the European Atomic Energy Community's (Euratom) Seventh Framework Program FP7/2007-2011 under the Project CHANDA (Grant No. 605203)

\section{References}

1. T. Goorley, "MCNP6.1.1-Beta Release Notes", LA-UR-14-24680 (2014).

2. Cetnar J. et al., MCB - a continuous energy Monte Carlo Burnup code, OECD/NEA, Fifth international information exchange meeting, Mol (1998).

3. A. Kochetkov et al., Current progress and future plans of the FREYA Project, Proceedings of the Second International Workshop on Technology and Components of Accelerator-driven Systems, Nantes, France 21-23 May 2013

4. H. A. Abderrahim ., MYRRHA a flexible and fast spectrum irradiation facility, Proceedings of the $11^{\text {th }}$ International Topical Meeting on Nuclear Applications of Accelerators (AccApp 2013), 5-8 August 2013, Bruges, Belgium

5. G. Ricco et al., Eur. Phys. J. Plus, 129 (2014).

6. G. Lomonaco et al. Eur. Phys. J. Plus, 129(74) (2014)

7. L. Mansani (Ansaldo Nucleare), private communication.

8. M. Osipenko et al. Eur. Phys. J. Plus, 129(68) (2014)
9. B. Brun and F. Rademakers, ROOT, An Object Oriented Analysis Framework, Proceedings AIHENP'96 Workshop, Lausanne, Sep. 1996, Nucl. Inst. \& Meth. in Phys. Res. A 389 (1997), https://root.cern.ch/

10. A. Borio di Tigliole, et al., Preliminary TRIGA Fuel Burn-up evaluation by means Monte Carlo code and computation based on total energy released during reactor operation, PHYSOR 2012, Knoxville, Tennessee, USA, April 15-20, 2012, on CDROM, American Nuclear Society, LaGrange Park, Illinois (2012). 